\title{
Carcinoma adenóide cístico de traquéia: tratamento pela laringotráqueo-esofagectomia e traqueostomia mediastinal*
}

\author{
Manoel Ximenes netto ${ }^{1}$, Gutemberg F. Rego ${ }^{2}$, André L.A. Carvalho ${ }^{3}$, Hélcio L. Miziara ${ }^{4}$
}

Os autores descrevem o caso de uma paciente de 54 anos de idade com carcinoma adenóide cístico de traquéia ao nível da cricóide, na qual foi realizada ressecção extensa, incluindo a laringe, parte da traquéia e esôfago. A reconstrução do trato digestivo foi através do tubo gástrico e a via aérea pela construção de uma

traqueostomia mediastinal. Cinco anos mais tarde foi removida uma recorrência tumoral cutânea no pescoço. Sete anos depois do procedimento original, foi notada nova recorrência no mediastino, que respondeu à irradiação. A paciente encontra-se muito bem atualmente, oito anos e cinco meses após 0 procedimento. (J Pneumol 1999;25(6):343-346)

\section{Adenoid cystic carcinoma of the trachea: treatment by removing the larynx, trachea, and esophagus, and mediastinal tracheostomy}

The authors describe the case of a 54 year-old female with adenoid cystic carcinoma of the trachea at the cricoid level upon whom extensive surgical resection was performed, including the larynx, part of the trachea and esophagus. Reconstruction of the digestive tract was done by means of a gastric tube and the airway with a mediastinal tracheostomy. Five years after the original procedure there was a

local cutaneous recurrence which was removed. Seven years later the disease recurred in the mediastinum, which responded to irradiation. The patient is alive and well eight years and five months after the operation.

Descritores - Traquéia. Neoplasma. Carcinoma adenóide cístico. Traqueostomia.

Key words - Trachea. Neoplasm. Carcinoma, adenoid cystic. Tracheostomy.

\section{INTRODUÇÃO}

A incidência de tumores traqueais é inferior a 0,2 por 100.000 por ano e tem prevalência de 1 pessoa para cada

* Trabalho realizado na Unidade de Cirurgia Torácica do Hospital de Base do Distrito Federal, Brasília, DF.

1. Livre-Docente; Chefe da Unidade de Cirurgia Torácica; Professor do Curso de Pós-Graduação da Universidade de Brasília; FACS, FICS, FCCP, TCBC.

2. Cirurgião de Tórax do Hospital da Unimed de Brasilia; Ex-Residente da Unidade de Cirurgia Torácica.

3. Cirurgião de Tórax do Hospital Universitário de Brasilia; Ex-Residente da Unidade de Cirurgia Torácica.

4. Chefe da Unidade de Anatomia Patológica.

Endereço para correspondência - Manoel Ximenes Netto, SHIN QI 07, Cj. 12, Casa 21 - 71515-120 - Brasilia, DF. Tel./ fax (061) 577-

4225; E-mail: Ximenes@cd-graf.com.br

Recebido para publicação em 20/3/99. Reapresentado em

2/8/99. A provado, após revisão, em 28/9/99.
Siglas e abreviaturas utilizadas neste trabalho

CAC - Carcinoma adenóide cístico

CEA - Antígeno carcinoembriônico

15.000 autópsias ${ }^{(1)}$. Levando em conta todas as mortes por câncer, esse tumor corresponde apenas a 0,1\%(2).

No grupo de tumores traqueais, a incidência do carcinoma adenóide cístico (CAC) varia de 18 a $59 \%$, vindo logo após o carcinoma epidermóide, que compreende, na maioria das publicações, metade de todas as neoplasias malignas da traquéia(3,4). Entre 1969 e 1990 (Medline), foram descritos e operados 153 pacientes portadores de carcinoma adenóide cístico de traquéia e, até junho de 1999, mais 283 pessoas foram tratadas de forma cirúrgica com essa patologia $a^{(1,2,4-10)}$. Devido à raridade dessa lesão, poucas instituições têm tido a oportunidade de adquirir experiência suficiente nesse tipo de neoplasia e, conseqüentemente, o manuseio fica restrito a alguns centros médicos especializados. A necessidade de ressecções extensas (exenteração cervical) nesse subgrupo de tumores traqueais é de $4,7 \%$ e, a mortalidade, de $5 \%^{(11)}$. A literatura médica latino-americana (Lilacs), em 216 registros de patologia traqueal, indexou quatro publicações referentes ao carcinoma adenóide cístico de traquéia, num total de sete casos, sendo seis no Brasil e um na Colombia ${ }^{(12-15)}$. 


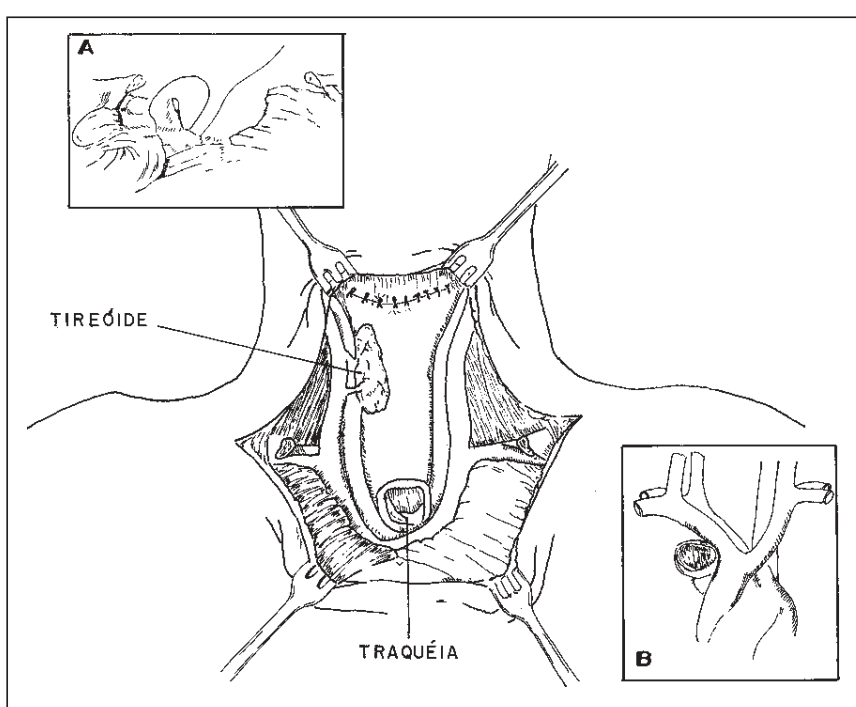

Figura 1 - Aspecto final do procedimento de ressecção de carcinoma adenóide cístico de traquéia. Em $\boldsymbol{A}$, sutura do músculo grande peitoral à porção remanescente do esterno posteriormente; em $\boldsymbol{B}$, posicionamento da traquéia restante, posteriormente à artéria inominada, a fim de evitar a erosão do vaso pela compressão. No centro, observa-se a tireóide restante e a sutura do tubo gástrico à faringe.

O objetivo do presente trabalho é relatar o caso de uma paciente com carcinoma adenóide cístico da traquéia submetida a extenso procedimento cirúrgico e que, apesar das recidivas, sobrevive por um período de quase nove anos.

\section{ReLATO dO CASO}

Mulher de 45 anos, queixava-se de disfagia e dor à deglutição, além de dispnéia aos médios esforços. Laringoscopia indireta demonstrou astenia de cordas vocais com pequena mobilidade em posição paramediana bilateral. U m esofagograma mostrou pequeno estreitamento na transição faringe/ esôfago, confirmado à esofagoscopia. No dia seguinte ao exame foi notada a presença de enfisema mediastinal e cervical, sugerindo perfuração. 0 pescoço foi explorado, drenado e iniciada antibioticoterapia. Tomografia computadorizada do pescoço mostrou aumento das partes moles entre a traquéia e o esôfago. Não havia adenomegalias, mas foi observado aumento de volume das veias jugulares direita e esquerda. Um mês depois, em virtude de dispnéia intensa, foi feita uma biópsia de lesão localizada no primeiro anel traqueal, envolvendo a região subglótica e esôfago cervical. 0 diagnóstico estabelecido pela biópsia foi de carcinoma adenóide cístico. Foi realizada uma traqueostomia abaixo da lesão e a paciente foi submetida aos procedimentos descritos em seguida.

\section{Técnica cirúrgica}

A técnica cirúrgica empregada para a remoção do tumor primário e os órgãos envolvidos consistiu inicialmente numa



Figura 2

Traqueostomia bem cicatrizada

incisão transversa supraclavicular até expor o músculo esternoclidomastóideo de forma bilateral, os quais em seguida foram seccionados. Estes últimos e o feixe carotídeo foram afastados lateralmente e a laringe e traquéia tracionadas para a linha média, posterior à fáscia pré-vertebral. U ma segunda incisão foi delineada na parede torácica, paralela à abertura cervical $10 \mathrm{~cm}$ abaixo. A fáscia do músculo grande peitoral foi dividida na linha média, expondo a primeira e segunda cartilagens costais e as respectivas costelas. 0 terço superior do esterno, além do terço médio de ambas as clavículas, foi seccionado, expondo a entrada superior do mediastino, e todos os gânglios subjacentes, além do timo e gordura mediastinal, dissecados e mobilizados juntamente com o tumor. A laringe, igualmente mobilizada, e a traquéia foram divididas de forma oblíqua. 0 tubo orotraqueal foi em seguido removido e a paciente reintubada na traquéia distal remanescente. 0 esôfago proximal foi amplamente mobilizado pelo leito mediastinal e o tubo preparado após laparotomia mediana ${ }^{(16)}$. 0 tubo gástrico ascendeu pelo leito esofagiano e a anastomose foi feita na altura da faringe. U m lobo da tireóide e a paratireóide subjacente foram preservados, sem prejudicar a radicalidade da operação. Os músculos peitorais foram suturados ao periósteo do esterno, posteriormente. A traquéia restante foi mobilizada distalmente, de modo a ser posicionada atrás da artéria inominada, para evitar tensão na linha de sutura com a pele e subseqüente erosão deste vaso (Figura 1). Um segmento de pele foi removido e em seguida suturado à traquéia (Figura 2). U ma jejunostomia alimentar também foi feita.

O estudo anatomopatológico da peça cirúrgica confirmou o diagnóstico de carcinoma adenóide cístico (Figura 3). Havia comprometimento da margem traqueal. Os gânglios não estavam comprometidos. 


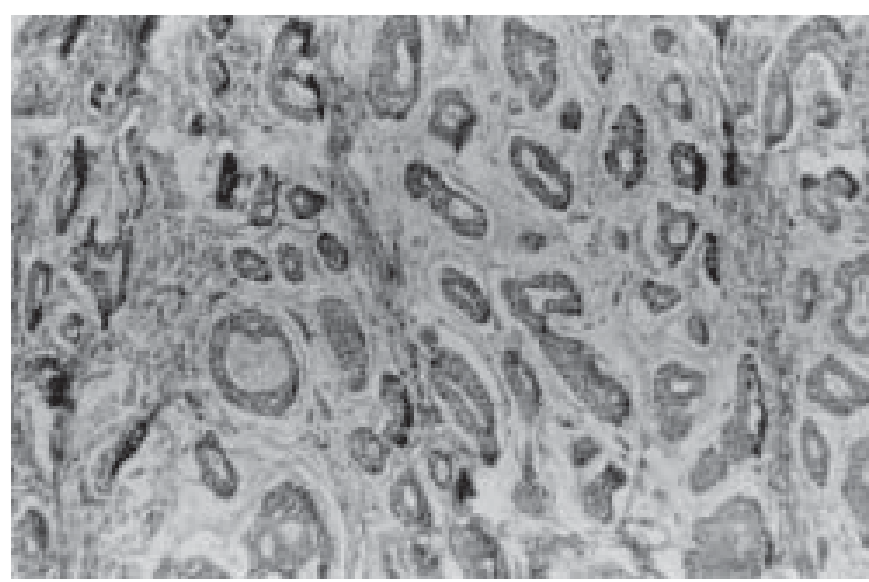

Figura 3 - A foto revela estrutura neoplásica formada por células epiteliais dispostas ora em túbulos ora com aspecto cribiforme (HE, 120x).

\section{Pós-operatório e complicações}

No pós-operatório imediato a paciente desenvolveu sinais de hipoparatireoidismo, o qual respondeu bem ao uso de cálcio e tiroxina. Os níveis hormonais têm-se mantido dentro da normalidade, bem como o cálcio e fósforo ( $\mathrm{T} 3=68$, $\mathrm{T} 4=6,2$ e TSH $=1,0$, cujos valores normais são de $80,0 \mathrm{a}$ $200,0,4,5$ a 12,5 e 0 a 4,0, respectivamente). Esofagograma mostrou boa passagem do contraste e a paciente ganhou cinco quilos de peso (Figura 4).

Q uatro anos depois, foi ressecada uma lesão cervical cutânea medindo $4,0 \times 2,0 \mathrm{~cm}$, correspondente à recidiva de tumor adenóide cístico e, após três anos, a paciente apresentou disfagia alta e estreitamento na altura da anastomose faringoesofagiana e na entrada do mediastino superior, correspondendo à recidiva da lesão. Esta área foi irradiada e feita uma gastrostomia. H ouve alívio completo da disfagia e o tubo de gastrostomia foi removido dois meses mais tarde.

A tualmente, a paciente se apresenta muito bem, oito anos e cinco meses após o procedimento original.

\section{DISCUSSÃO}

O carcinoma adenóide cístico é um tumor raro da via aérea superior que permite sua ressecção às vezes de forma radical, incluindo a lesão primária, além de todas as estruturas locais envolvidas e reconstrução das vias aérea e digestiva, com morbidade e mortalidade aceitáveis ${ }^{(17,18)}$. Os resultados a longo prazo de séries, tanto na Europa como na América do Norte, estão no Quadro 1.

Esse tipo de tumor costuma difundir-se além da margem de ressecção cirúrgica, que pode apresentar-se grosseiramente normal, e através dos linfáticos da submucosa e perineurais. Mesmo assim, havendo comprometimento da margem de ressecção, os resultados são favoráveis. A mortalidade na experiência do grupo de Toronto foi de $9 \%$ em 37 casos de carcinoma adenóide cístico ${ }^{(5)}$. Na vivência do Massachusetts

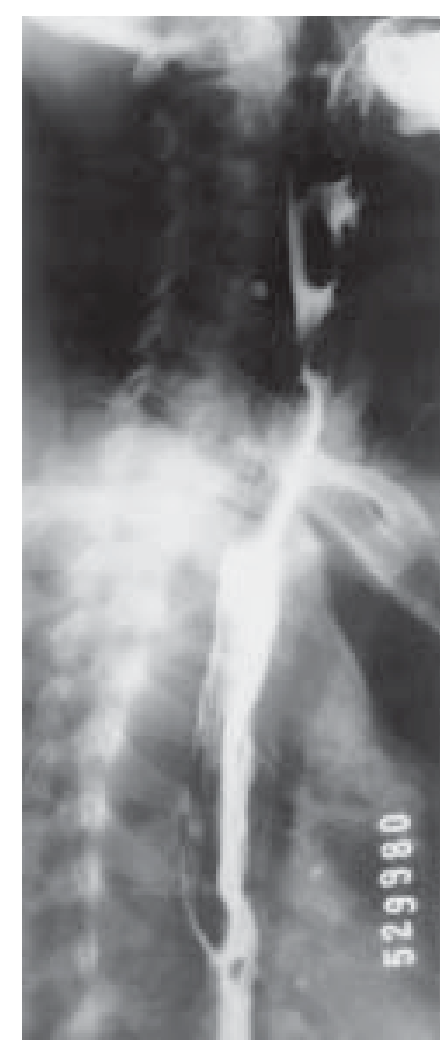

Figura 4 - Esofagograma mostrando o tubo gástrico usado na substituição do esôfago e anastomosado à faringe

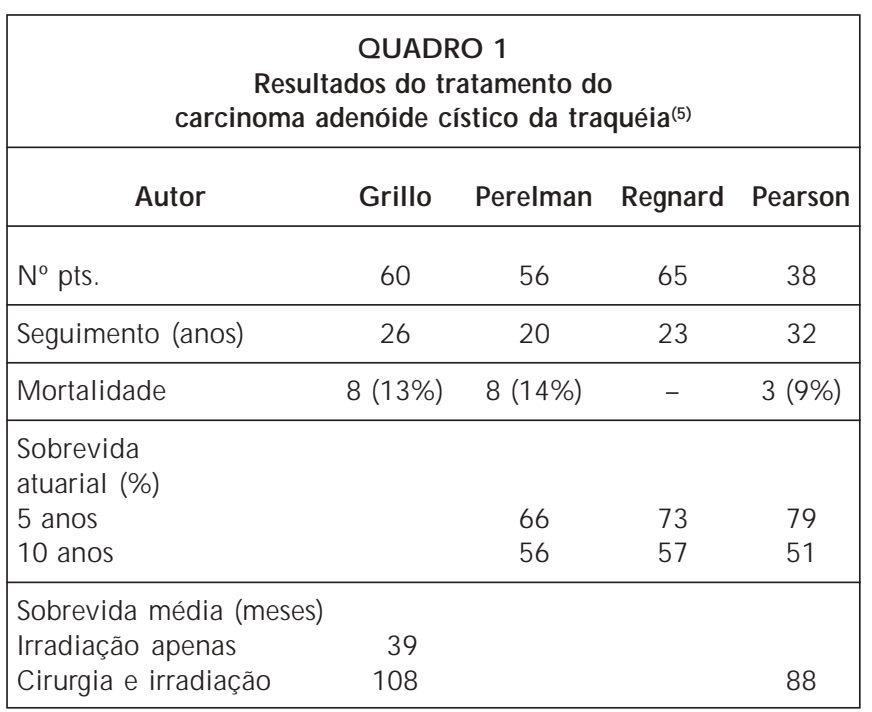

General Hospital com a exenteração cervical para esse tipo de tumor, não houve mortalidade em três pacientes e a morbidade, incluindo todos os 18 pacientes da série, foi elevada. Os problemas relatados foram separação do estoma traqueal em dois, hemiplegia do lado direito em um, pneumonia em três e trombose ileofemoral em outro. Hormônio tireoidiano, vitamina $D$ e cálcio foram administrados em 11 pacientes ${ }^{(19)}$. 
A radioterapia, tanto adjuvante como pós-operatória, é recomendada, especialmente nos casos de recidiva. Por outro lado, não existe nenhuma evidência de que a quimioterapia exerça qualquer atividade benéfica nesse tipo de tumor ${ }^{(20)}$. A radioterapia apenas, nos casos considerados irressecáveis, ainda pode oferecer sobrevida razoável, acima de seis anos, na experiência de Pearson ${ }^{(5)}$. Metástases linfáticas são incomuns, e, quando presentes, não alteram o prognóstico nas diversas séries relatadas $(6,7,11,21)$. A possibilidade de monitorar o carcinoma adenóide cístico da traquéia através do CEA (antígeno carcinoembriônico) foi demonstrada em um paciente em que foi medida essa glicoproteína oncofetal(22). Tratamento

\section{REFERÊNCIAS}

1. Azar T, Abdul-Karim FW, Tucker HM. Adenoid cystic carcinoma of the trachea. Laryngoscope 1998;108:1297-1300.

2. Pearson FG, Cardoso $P$, Keshavjee S. Primary tumors of the upper airway. In: Pearson FG, Deslauries J, Ginsberg RJ, eds. Thoracic surgery. 1st ed. New York: Churchill \& Livingstone, 1995;285-299.

3. Li W, Ellerbroeck NA, Libshitz HI. Primary malignant tumors of the trachea. A radiological and clinical study. Cancer 1990;66:894-899.

4. Refaely $Y$, Weissberg $D$. Surgical management of tracheal tumors. Ann Thorac Surg 1997;64:1429-1432.

5. Maziak DE, Todd TRJ , Keshavjee SH, et al. Adenoid cystic carcinoma of the airway: thirty-two-year experience. J Thorac Cardiovasc Surg 1996;112:1522-1537.

6. Perelman MI, Koroleva N, Birjokov J, Goudovsky L. Primary tracheal tumors. Semin Thoracic Cardiovasc Surg 1996;8:400-402.

7. Regnard J F, Fourquier P, Levasseur P. Results and prognostic factors in resections of primary tracheal tumors: a multicenter retrospective study. The French Society of Cardiovascular Surgery. J Thorac Cardiovasc Surg 1996;111:808-813.

8. Prommegger R, Salzer GM. Long-term results of surgery for adenoid cystic carcinoma of the trachea and bronchi. Eur J Surg Oncol 1998; 24:440-444.

9. Hartley BE, Fagan J J Rhys-Evans PH. Excision of adenoid cystic carcinoma of the cervical trachea via an anterior castellated approach. J Laryngol Otol 1995;8:774-776.

10. Sur RK, Pacella J A, Levin V. A denoid cystic carcinoma of the trachea. A report of two cases. S Afr J Surg 1996;34:191-192.

11. Grillo HC, Mathissen DJ. Primary tracheal tumors: treatment and results. Ann Thorac Surg 1990;49:69-77. paliativo através do laser endoscópico e irradiação também foi descrito em um caso com sobrevida de nove anos ${ }^{(23)}$.

O carcinoma adenóide cístico da via aérea alta, em resumo, é uma lesão que ocorre na idade adulta, cresce devagar, permite ampla ressecção, seja da via aérea apenas ou de outras estruturas envolvidas, com baixa mortalidade e morbidade. A maioria responde muito bem à irradiação, tanto nas recidivas como na terapia adjuvante, especialmente nos casos em que ocorre comprometimento da margem cirúrgica. Bons resultados são esperados, tanto nas ressecções completas, quanto nas incompletas.

12. Forte $\mathrm{V}$. O que mudou no tratamento dos tumores da traquéia nas últimas décadas. Rev Bras Cir Cabeça e Pescoço 1987;11:11-22.

13. Panain VLN, A vila CM, Lagreca HR, Cunha FC. Carcinoma adenóide cístico da traquéia. Folha Med 1987;94:91-93.

14. Cardoso JJD, Alvim L, Capella N, Souto AF. Tumor carcinóide de traquéia. ACM Arq Catarin Med 1994;23:251-252.

15. Ramirez J C, Pelaez M, Rincón J D. Reseccion y reconstruccion de la carina. Rev Colomb Neumol 1997;9:271-278.

16. Ximenes Netto M, Silva RO, Vieira LF, Canindé MLC. Esophageal replacement with a isoperistaltic gastric tube. Rev Goiana Med 1984; 3:111-119.

17. Grillo HC. Tracheal tumors: surgical management. Ann Thorac Surg 1978;26:112-125.

18. Larrson S, Cardillo G, Lepore V. Surgical management of tracheal tumours. Scand J Thorac Cardiovasc Surg 1987;21:97-103.

19. Grillo HC, Mathisen DJ . Cervical exenteration. Ann Thorac Surg 1990; 49:401-409.

20. Pearson FG, Thompson DW, Weissberg D, et al. A denoid cystic carcinoma of the trachea. Ann Thorac Surg 1974;18:16-29.

21. Gelder CM, Hetzel MR. Primary tracheal tumors: A national survey. Thorax 1993;48:688-692.

22. Kuhel WI, Chow H, Godwin TA, Minick CR, Libby RM. Elevated carcinoembryonic antigen levels correlating with disease recurrence in a patient with adenoid cystic carcinoma. Head Neck 1995;17:431-436.

23. Okahara M, Segawa $Y$, Takigawa $N$, et al. Primary adenoid cystic carcinoma of the trachea effectively treated with endoscopic Nd-YAG laser followed by irradiation. Ann Int Med 1996;35:146-149. 